\title{
Effect of proanthocyanidin on push-out bond strength between fiber post and root dentin- An in-vitro study
}

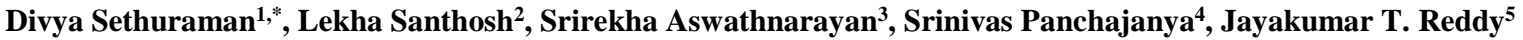 \\ ${ }^{1}$ Post graduate Student, ${ }^{2,5}$ Professor, ${ }^{3}$ Professor \& HOD, ${ }^{4}$ Reader, Dept. of Conservative Dentistry and Endodontics, The Oxford \\ Dental College, Bengaluru, Karnataka, India
}

*Corresponding Author:

Email: sethuraman.divya@gmail.com

\begin{abstract}
Aim: The aim of this study was to assess the influence of Proanthocyanidin (PA) application on bond strength of glass fiber posts to root canal dentin using self-etch adhesive luting system.

Materials and Methods: Twenty single rooted mandibular premolars, freshly extracted for orthodontic purpose, were decoronated below the cementoenamel junction, and standardized to $14 \mathrm{~mm}$ in length. Post space preparation was done upto No 3 Peeso reamer during which canals were irrigated with conventional irrigation protocol. Teeth were randomly divided into two groups ( $\mathrm{n}=20$ each)

Group 1: Pre-treatment with PA and Group 2 served as the control group. Both Groups were restored with fiber posts bonded with RELYX U200 self-etch adhesive system. Teeth were then stored in distilled water for three months at $37^{\circ} \mathrm{C}$. One mm slices were obtained and push-out bond strength testing was performed. Failure patterns were assessed under stereomicroscope at 20x magnification.

Results: Individual bond strength values were calculated and statistically analysed with the chi-square test and independent-ttest. A statistically significant difference existed between the two Groups $(\mathrm{p}<0.001)$ with Group 1 showing a mean bond strength value greater than Group 2.

Conclusion: Pretreatment with PA preserves the bond strength of fiber posts to root dentin bonded with a self-etch adhesive system. The results indicate that PA can be used as a biocompatible pretreatment alternative to improve bond strength stability of dentin-adhesive interfaces in root canals.
\end{abstract}

Keywords: Collagen-crosslinking, Glass fiber post, MMP inhibitor, proanthocyanidin, push-out bond strength, self-etch adhesive system.

\section{Introduction}

Endodontically treated teeth often lose substantial tooth structure and hence the need for a post that reinforces remaining tooth structure arises. ${ }^{1,2}$ Nonmetallic fiber posts improve stress distribution because their modulus of elasticity is similar to that of dentin. ${ }^{3}$ Durability of the bond between fiber posts and root dentin is an important issue for providing long-term clinical success. ${ }^{4}$

Proanthocyanidins (PAs) are a group of bioflavanoid compounds which promote cross-linking of collagen and enhance the strength of the resin-dentin interface when used as an external collagen crosslinking agent. ${ }^{5-9}$

The everStick system is an uncured flexible fiber post that works on the interpenetrating polymer network (IPN) technology. Till date no study has evaluated the effect of PA on bond strength of everStick fiber post system to root dentin.

Hence the aim of this study was to assess the influence of PA application on bond strength of flexible glass fiber posts (everStick) to root dentin using selfetch adhesive luting system.

\section{Materials and Methods}

Twenty freshly extracted single-rooted human mandibular premolar teeth, extracted for orthodontic purpose, with anatomically similar root segments and fully developed apices were selected. Teeth with previous endodontic treatment, cracks or calcified canals were excluded. Teeth were stored in $0.1 \%$ thymol solution until use. Each tooth was decoronated below the cemento-enamel junction perpendicular to the longitudinal axis using a slow-speed, water-cooled diamond disk such that they were cut to a uniform length of $14 \mathrm{~mm}$ from the apical end. Only teeth with round canal cross-section were selected. The endodontic access cavity was refined using a \#2 ENDO ACCESS bur (Denstply, Maillefer).

Pulp remnants was removed using files \#10K and \#15K file (Mani. Inc Japan). Peeso reamers (Mani. Inc Japan) sizes 1, 2 and 3 (ISO size70 and 90 and 110 respectively) were used to prepare the post space of $10 \mathrm{~mm}$ length into which an everStick fiber post of diameter $1.2 \mathrm{~mm}$ was to be fitted. The canals were irrigated during post space preparation followed by a final irrigation protocol of $5 \mathrm{ml}$ each of $3 \%$ sodium hypochlorite (Vensons India), 17\% EDTA solution (Pulpdent EDTA solution, Pulpdent Corporation) and $0.9 \%$ normal saline.

Following post space preparation, the teeth were randomly divided into two groups $(n=20)$ : 
Group 1: Root canals treated with PA and restored with glass fiber post

Group 2: Non-PA treated root canals restored with the glass fiber post

Preparation of PA solution: For each tooth specimen freshly prepared experimental solution was obtained by dissolving 95\% PA (Perennial Life sciences Privates Limited, Delhi) in distilled water to obtain $6.5 \%$ solution $(0.4675 \mathrm{gm}$ of PA was dissolved in $12.5 \mathrm{ml}$ of distilled water to get the required concentration).

Group 1: The PA solution (1ml) was applied to the prepared post space in the root canal with a 27 -gauge open ended needle. Care was taken to ensure the absence of air bubbles and the solution was left in place for 1 minute after which it was blot-dried using paper points.

Post placement: A dual-polymerizing self-etch adhesive resin luting material (RelyX, 3M INDIA) was injected into the prepared root canal space. Subsequently, the fiber post was covered with cement, seated inside the root canal and kept under finger pressure for 20 seconds and the excess cement was removed. The cement and post were polymerised for 40 seconds (LEDition ${ }^{\mathrm{R}}$, Ivoclar Vivadent Inc.,) according to manufacturer's instructions.

Group 2: The teeth in this group were restored with fiber post in the same way as in Group1 except for pretreatment with PA.

Specimens of both the groups were stored in distilled water which was changed periodically with a fresh supply once every four days. This was done for a period of 3 months at $37^{\circ}$ Celsius in an incubator (Avilab, India) after which they were subjected to pushout bond strength test.

\section{Results}

Table 1: Comparison of Groups 1 and 2 with respect to bond strength in MPa by independent $t$ test

*Significant difference

\begin{tabular}{|l|l|l|l|l|l|l|}
\hline Groups & n & Mean & SD & SE & t-value & p-value \\
\hline Group 1 & 20 & 3.51 & 0.08 & 0.02 & 44.0971 & $<0.001^{*}$ \\
\hline Group 2 & 20 & 2.39 & 0.08 & 0.02 & & \\
\hline
\end{tabular}

Table 2: Comparison of failure modes between Groups 1 and 2

\begin{tabular}{|c|c|c|c|c|c|c|c|}
\hline Failure mode & Group 1 & \% & Group 2 & \% & Total & \% \\
\hline 1 & 2 & 10.00 & 11 & 55.00 & 13 & 32.50 \\
\hline 2 & 0 & 0.00 & 5 & 25.00 & 5 & 12.50 \\
\hline 3 & 10 & 50.00 & 0 & 0.00 & 10 & 25.00 \\
\hline 4 & 8 & 40.00 & 0 & 0.00 & 8 & 20.00 \\
\hline 5 & 0 & 0.00 & 4 & 20.00 & 4 & 10.00 \\
\hline & 20 & 100.00 & 20 & 100.0 & 40 & 100.00 \\
\cline { 2 - 8 } & & & & & & \\
\hline
\end{tabular}

*Highly significant difference
Each root was cut horizontally with a rotary microtome (Physilab, Germany) into $1 \mathrm{~mm}$ sections. Sections were obtained from cervical third and middle third of the root.

Push-out test and Failure mode analysis: The pushout test was performed by applying a load at $1 \mathrm{~mm} / \mathrm{min}$ in an apico-coronal direction until the fiber post segment was dislodged from the root slice. Care was taken to make sure the punch tip was centralized in the root canal and positioned to contact only the post/cement without stressing the surrounding root canal walls. The push-out bond strength was measured with a universal testing machine (Multitest-i, Mecmesin, England). To express the bond strength in megapascals $(\mathrm{MPa})$, the load at failure recorded in newtons $(\mathrm{N})$ was divided by the area $\left(\mathrm{mm}^{2}\right)$ of the postdentin interface. To calculate the bonding area, the formula

$22 / 7(\mathrm{R}+\mathrm{r})\left[(\mathrm{h})^{2}+(\mathrm{R}-\mathrm{r})^{2}\right]$, was used, where ' $\mathrm{R}$ ' represents the coronal root canal radius, ' $r$ ' the apical root canal radius and ' $h$ ' the thickness of the slice. The thickness of each slice was measured using a digital caliper.

The de-bonded specimens were observed under 20x magnification with a stereomicroscope (ZOOMAR, Lawrence \& Mayo) to classify the failure pattern into 5 types according to Cecchin et al. ${ }^{9}$

The data for the two Groups were tabulated on a Microsoft Excel sheet and subjected to statistical analysis with independent-t test and chi square test. The level of significance was set at $<0.05$. 


\section{Discussion}

In today's era of dentistry that focuses widely on improved adhesion in the root canal, resin cements are used for bonding the fiber post systems. Maintaining the structural integrity of the hybrid layer against hydrolytic and enzymatic degradation is of prime importance and is an important issue for providing long-term clinical success. ${ }^{5}$ Dentin adhesion creates a resin-enveloped collagen scaffold that, ideally will remain in place for decades. ${ }^{10}$ Nevertheless, the hybrid layer may be degraded over time, leading to failure of the adhesive interface. ${ }^{11,12}$

Previous studies have shown that the application of exogenous crosslinking agents to several connective tissues is helpful to modify the structures of collagen fibrils, and improve their degradation resistance as well as stabilization by inhibition of MMPs. ${ }^{5-9,13-17}$ One recently appreciated cross-linking agent, PA belongs to a class of bioflavonoids that are naturally occurring plant metabolites available in fruits, vegetables etc. This agent consequently increases the mechanical properties of root canal dentin collagen, enhances biodegradation resistance and improves bond durability. ${ }^{5}$

An electrical glass fiber post system, ever Stick post (GC, India), is available in an uncured flexible form and hence can be adapted to take up the morphological shape of the root canal.

This system has a patented IPN which has upto 4000 fibers fully impregnated with resin. This fusion of fibers and resin forms an exceptionally strong, esthetic and user-friendly system.

Yoshida et al revealed that the mild acidic monomers on the self-etch systems (RelyXU200, 3M, India) are not aggressive enough to partially demineralize the intact dentin surface, even in the absence of a smear layer. ${ }^{18}$ Hence $17 \%$ EDTA as an irrigating solution was also used to remove the smear layer and enhance formation of a hybrid layer with selfetch adhesive system.

The results of this study showed a significant difference $(\mathrm{p}<0.001)$ in the mean bond strength values between the two groups. Thus the null hypothesis was rejected. The application of mildly acidic self-etch adhesive activates MMPs, resulting in progressive proteolytic degradation of exposed collagen fibrils within the hybrid layer after storage period of 3 months. On the contrary in Group 1, application of PA led to collagen cross-linking, which may have contributed to higher bond strength values.

A study ${ }^{9}$ done with $6.5 \%$ PA but using a regular fiber post and assessing 12 months water storage period gave mean bond strength values which corroborated with the present study. PA consists of highly hydroxylated structures that are capable of forming insoluble complexes with carbohydrates and proteins. The higher stability of adhesive interfaces treated with
PA can also be explained by the inhibition of proteolytic activity of enzymes. However, the specific mechanism of inhibition of proteolytic enzymes by PAs is unknown. Changes in the conformation of bacterial collagenase promoted by PAs through hydrogen and hydrophobic interactions have been suggested. ${ }^{19}$ For MMPs, cross-linkage of the catalytic sites of these enzymes and inhibition of other non-collagenous proteins that activate these enzymes also have been hypothesized. ${ }^{20,21}$ Possibly, the combined cross-linking and anti-collagenolytic effects of PAs are responsible for preventing degradation of dentin collagen within the hybrid layer. ${ }^{8}$

Results of the failure analysis by chi-square test, showed that a highly significant difference existed in between Groups $1 \& 2$ ( $p<0.0001)$. In Group 2, failure mode 3 , i.e. mixed failure with resin cement covering $50 \%-100 \%$ of the post's surface was seen in maximum number of specimens $(50 \%)$. This indicates that the resin-dentin interface was weak, which led to an adhesive failure.

In Group 1, Failure 1, i.e. Adhesive failure, between the fiber post and resin cement (no cement visible around the post) was found to be the most predominant $(55 \%)$. This could be graded as one of the most favourable outcomes of the study as an adhesive failure between the fiber post and resin cement simply indicates that the resin-dentin interface had a superior bond which probably led to the resin cement adhering to the root canal dentin surface as suggested by Kalra et al. ${ }^{6}$

Apart from the two types of adhesive failure, another noticeable failure mode occurred in $20 \%$ of the specimens in Group 1. This was Failure 5, which was a cohesive failure in dentin. The superior bond strength at the dentin-resin interface after the treatment with PA may have caused the dentin to fracture.

Further studies that evaluate longer storage time and/or simulated aging are encouraged. The post surfaces that were studied under the stereomicroscope were found to have a brownish hue which was probably acquired from the color of the PA solution. Hence, studies that assess the effect of staining from the PA solution have to be encouraged.

\section{Conclusion}

Within the limitations of the present study, it can be concluded pretreatment with PA strengthens the bond strength of flexible fiber posts significantly to root dentin with the use of a self-etch adhesive system after a storage period of 3 months. PA can be used as a biocompatible pretreatment alternative to improve bond strength stability of dentin-adhesive interfaces in root canals.

\section{References}

1. Sherfudhin H, Hobeich J, Carvalho CA, Aboushelib MN, Sadig W, Salameh Z. Effect of different ferrule designs 
on the fracture resistance and failure pattern of enododontically treated teeth restored with fiber posts and all-ceramic crowns.J Appl Oral Sci 2011;19:28-33

2. Toman M, Toksavul S, Tamac E, Sarikanat M, Karagozoglu I. Effect of Chlorhexidine on bond strength between glass-fiber post and root canal dentin after six month of water storage. Eur J Prosthodont Restor Dent2014;22:29-34

3. Pest LB, Cavalli G, Bertani P, Gagliani M. Adhesive post-endodontic restorations with fiber posts: push-out tests and SEM observations. Dent Mater 2002; 18:596602.

4. Sudsangiam S, Van Noort R. Do dentin bond strength tests serve a useful purpose? J Adhes Dent 1999;1:57-67.

5. Kalra M, Iqbal K, Nitisusanta LI, Daood U, Sum CP, Fawzy AS. The effect of proanthocyanidins on the bond strength and durability of resin sealer to root dentine. Int Endod J 2013;46:169-78.

6. Nagpal R, Singh P, Singh S, Tyagi SP. Proanthocyanidin: A natural dentin biomodifier in adhesive dentistry. J Res Dent 2016;4:1-6.

7. Manimaran VS, Srinivasulu S, Rajesh Ebenezer A, Mahalakshmi S, Srinivasan N. Application of a proanthocyanidin agent to improve the bond strength of root dentin treated with sodium hypochlorite. J Conserv Dent 2011;14:306-8.

8. Sakhamuri S, Sampath V, Manimaran V, Mahalaxmi S. Effect of collagen cross-linkers on the shear bond strength of a self-etch adhesive system to deep dentin. J Conserv Dent 2013;16:135-8.

9. Cecchin D, Pin LC, Farina AP, Souza M, Vidal Cde M, Bello YD et al. Bond strength between fiber posts and root dentin treated with natural cross-linkers. J Endod 2015;41:15.

10. Jainaen A, Palamara JEA, Messer HH. Push-out bond strengths of the dentin-sealer interface with and without a main cone. Int Endod J 2007;40:882-90

11. Spencer P, Ye Q, Park J, Topp EM, Misra A, Marangos O. Adhesive/dentin interface : the weak link in composite restoration. Ann Biomed Eng. 2010 Jun;38(6):1989-2003.

12. Hashimoto M, Ohno H, Sano H, Kaga M, Oguchi H. In vitro degradation of resin-dentin bonds analyzed by micro tensile bond test, scanning and transmission electron microscopy. Biomaterials. 2003 Sep;24(21):3795-803.

13. Hagerman AE, Klucher KM. Tannin-protein interactions. Prog Clin Biol Res1986;213:67-76.

14. Epasinghe DJ, Yiu CK, Burrow MF, Tay FR, King NM. Effect of proanthocyanidin incorporation into dental adhesive resin on resin-dentine bond strength. J Dent. 2012 Mar;40(3):173-80.

15. Green B, Yao X, Ganguly A, Xu C, Dusevich V, Walker MP et al. Grape seed proanthocyanidins increase collagen biodegradation resistance in the dentin/adhesive interface when included in an adhesive.J Dent. 2010 Nov;38(11):908-15.

16. Al-Ammar A, Drummond JL, Bedran-Russo AK. The use of collagen cross-linking agents to enhance dentin bond strength. J Biomed Mater Res B Appl Biomater. 2009 Oct;91(1):419-24.

17. Liu Y, Chen M, Yao X, Xu C, Zhang Y, Wang Y. Enhancement in dentin collagen's biological stability after proanthocyanidins treatment in clinically relevant time periods. Dent Mater. 2013 Apr;29(4):485-92.

18. 18. Yoshida Y, Nagakane K, Fukuda R, Nakayama Y, Okazaki M, Shintani H, et al. Comparative study on adhesive performance of functional monomers. J Dent Res 2004;83:454-8.
19. Bedran-Russo AK1, Pashley DH, Agee K, Drummond JL, Miescke KJ. Changes in stiffness of demineralized dentin following application of collagen cross-linkers. J Biomed Mater Res B Appl Biomater. 2008 Aug;86(2):330-4.

20. Yi Liu, Mingsheng Chen, Xiaomei Yao, Changqi Xu, Ying Zhang, Yong Wang Enhancement in dentin collagen's biostability after Proanthocyanidin treatment in clinically relevant time periods. Dent Mater. 2013 Apr;29(4):485-92.

21. Rui-Rui Liu, Ming Fang, Ling Zhang, Cheng-Fang Tang, Qi Dou, Ji-Hua Chen Anti-proteolytic capacity and bonding durability of PA biomodified demineralized dentin matrix. Int J Oral Sci. 2014 Sep;6(3):168-74. 